\title{
PERFORMANCE AND NEW CAPABILITIES OF SPRING-8
}

\author{
H.Kamitsubo, N.Kumagai, SPring-8/JASRI, Mikazuki, Hyogo, Japan
}

\begin{abstract}
SPring-8 was commissioned in March 1997 and put into the scheduled operation in October 1997. In 1998 the storage ring was operated for 4190 hours, $62.6 \%$ of which was the user time. Presently sixteen beamlines are operational in the user service mode, five are in commissioning and seventeen are under construction. We found that specifications of the accelerators exceed the design values and it becomes clear that the storage ring has much higher performance than that we had expected in the design phase. In addition, insertion devices developed at SPring- 8 have proved to be of high performance. In 1998 construction of a 30m long insertion device and beamline is started.
\end{abstract}

\section{OVERVIEW OF THE SPRING-8 FACILITY}

SPring- 8 is the third generation synchrotron radiation source in an energy range from 0.3 to several hundreds $\mathrm{keV}$. It is composed of a $1 \mathrm{GeV}$ injector linac, an $8 \mathrm{GeV}$ booster synchrotron and an $8 \mathrm{GeV}$ storage ring with a natural emittance of $7 \mathrm{nmrad}$. SPring- 8 is the national user facility enacted by the special law and is open to users not only in Japan but also from abroad.

Design studies of SPring- 8 and related R\&D works started in 1987. The project was decided to be promoted jointly by Japan Atomic Energy Research Institute (JAERI) and the Institute of Physical and Chemical Research (RIKEN) and the Joint Project Team was formed in 1988. The construction of the facility started in 1990 and the linac and the synchrotron were completed in August and in Decenber, 1996, respectively. Commissioning of the storage started in the middle of March and the first synchrotron radiation from a bending magnet was observed on March 23 1997. After the Dedication Ceremony held on October 6, 1997, SPring-8 entered into the scheduled operation in the user service mode [1].

The storage ring consists of 48 cells with a lattice structure of Chasman-Green type and has a circumference of $1,436 \mathrm{~m}$. Four of them are straight cells which are to be used for accommodation of very long insertion device. Presently the straight cell has the same arrangement of focussing magnets as the normal cell but no bending magnet. In this year, however, the construction of a beamline from a long straight section starts and beamdynamical study to modify the magnet arrangement to realize the dispersion-free long straight sections.

Besides the long straight sections the storage ring has 40 dispersion-free straight sections of $6.5 \mathrm{~m}$ long. One of them is used for beam injection and four are used for RF stations, each of which is composed of eight single-cell cavities of 508.6MHz. Furthermore one straight section is reserved to install harmonic cavities in future. The remaining 34 are used to accommodate standard-size insertion devices (ID). Total number of the beamlines is 62,38 of which are ID beamlines and 23 are bendingmagnet (BM) beamlines. There is one beamline for infrared spectroscopy.

The beamlines are classified into three categories; the public, the contract and the JAERI and RIKEN beamlines. Presently sixteen beamlines are operational, five are in commissioning, and seventeen are under construction.

The linac delivers $1 \mathrm{GeV}$ electrons to a $1.5 \mathrm{GeV}$ synchrotron/storage ring (New SUBARU), which is built by Himeji Institute of Technology and Hyogo Prefecture for their own use.

\section{PERFORMANCE OF SPRING-8}

Since the commissioning of the storage ring in March 1997, machine studies have been intensively pursued and it becomes clear that the storage ring is extremely stable and has high performance. Design values of the storage ring specifications are shown in Table 1 together with the achieved values[2].

\subsection{Operation of SPring-8}

Since October 1997 the storage ring has been operational in user service mode. As we had to construct more than 15 new beamlines in the past one year, we had shut down the machines in January, July and August 1998 to install their ID's and front end parts. Nevertheless we could operate the storage ring for 4190 hours in 1998 and provided photon beam to users for 2600 hours. In Table 2 is summarized the statistics of the storage ring operation in 1997 and 1998.

Presently we operate SPring- 8 continuously in three weeks (one cycle). In one cycle we start the operation of the machines in Wednesday morning and usually spend one day for machine and beamline tuning and, if it is possible, machine studies. User time starts in Thursday afternoon and ends in Wednesday evening of the third week. Then machine group uses two days for machine studies. The cycle just after a long shutdown of the machines is used only for tuning of machine and new beamlines.

Currently the storage ring is operated in several filling modes, that is, a full-filling, a several-bunch and a hybrid modes. More than $62 \%$ of the user time were delivered in a full-filling mode in 1998. Recently the storage ring is operated in a 2/3-filling mode for user time. 
The lifetime depends on the filling mode. At present beam lifetime at $70 \mathrm{~mA}$ is $90 \mathrm{hrs}$ in a full-filling mode and $56 \mathrm{hrs}$ in the $2 / 3$ filling mode. The reason why the beam lifetime is so long in a uniformly filling mode is that the electron beam size is grown by the instability due to an ion-trapping effect. This means that the beam quality in the full-filling mode is a little worse than that in the $2 / 3$ filling modes so that we adopt the $2 / 3$ filling mode for the routine operation.

Table 1: Performance of SPring-8 Storage Ring

\begin{tabular}{|l|c|c|}
\hline & Designed & Achieved \\
\hline Energy & $8 \mathrm{GeV}$ & $8 \mathrm{GeV}$ \\
\hline Circumference & $1435.948 \mathrm{~m}$ & \\
\hline $\begin{array}{c}\text { No. of Cells } \\
\text { (Normal / Straight) }\end{array}$ & $44 / 4$ & \\
\hline $\begin{array}{c}\text { Current(Single bunch) } \\
\text { (Multi-bunch) }\end{array}$ & $\begin{array}{c}5 \mathrm{~mA} \\
100 \mathrm{~mA}\end{array}$ & $12 \mathrm{~mA}$ \\
\hline Harmonic Number & 2436 & \\
\hline Revolution Time & $4.79 \mu \mathrm{mA}$ \\
\hline Tunes (vx/vy) & $51.22 / 16.16$ & $51.16 / 16.31$ \\
\hline Chromaticities ( $\xi_{\mathbf{x}} / \xi_{\mathrm{v}}$ ) & & $3.21 / 3.93$ \\
\hline Energy Spread $(\Delta \mathrm{E} / \mathrm{E})$ & $0.11 \%$ & $0.12 \%$ \\
\hline Emittance & $7 \mathrm{~nm} . \mathrm{rad}$ & $6.8 \mp 0.4 \mathrm{nmrad}$ \\
\hline Coupling & $<10 \%$ & $0.06 \%$ \\
\hline Life Time at 100mA & $>10 \mathrm{hr}$ & $\sim 50 \mathrm{hr}$ \\
\hline BunchLength (FWHM) & $34.2 \mathrm{ps}$ & $\sim 40 \mathrm{ps}$ \\
\hline $\begin{array}{c}\text { Dispersion (Vertical) } \\
\text { (Horizontal) }\end{array}$ & & $<0.01 \mathrm{~m}$ \\
(Vertical) & & $0.05 \sim 0.1$ \\
\hline COD Horizontal) & & $0.05 \sim 0.1$ \\
\hline
\end{tabular}

For experiments using the time structure of the beam the storage ring is operated in the several bunch mode. Filling patterns of this mode are equally-spaced single bunches or several-bunch trains. In this case bunch purity is routinely better than $10^{-6}$. Filling pattern of the hybrid mode is a mixture of equally-spaced single bunches and a long train of bunches.

The bunch length depends on the bunch current, too. We measured bunch width by a streak camera. The measured value is $40 \mathrm{ps}(\mathrm{FWHM})$ at a current lower than $1 \mathrm{~mA} /$ bunch and, due to inductive impedance of vacuum elements such as bellows and so on, increases rapidly with the bunch current.

\subsection{Emittance and Coupling}

The size of the stored electron beam in the horizontal plane was measured directly in the following way: The beam is put in the bump orbit by the DC bump magnet in the injection section and then deflected by the pulse bump magnet. The amplitude of the bump orbit changes with the field strength of the DC bump magnet and a part of the beam is lost by a septum wall of the septum magnet.
Table 2: Operation Statistics of SPring-8 (October 1997 to December 1998)

\begin{tabular}{|l|c|c|}
\hline & $\mathbf{1 9 9 7}$ & $\mathbf{1 9 9 8}$ \\
\hline Total Beam Time & $1290(\mathrm{hrs})$ & $4190(\mathrm{hrs})$ \\
\hline User Time* & 980 & 2624 \\
\hline Tuning \& Machine Studies** & 287 & 1456 \\
\hline Loss Time due to Failures & 26 & 110 \\
\hline MTBF $^{\#}$ & 43.5 & 43.7 \\
\hline
\end{tabular}

* does not include loss time due to failures

** includes time for new beamline commissioning

\# mean time between failures

Horizontal charge distribution was obtained from the relation between the beam loss rate and the bump orbit. Then the horizontal emittance of the beam was determined from the width of the horizontal charge distribution and a measured beta function. The measured value of the emittance is $6.8 \mathrm{nmrad}$.

The emittance in the vertical plane depends on the coupling between the horizontal and vertical betatron oscillations. In case of the SPring- 8 storage ring a skew component of the focussing magnets is very small so that the coupling is induced mainly by the nearest tune resonance of $v_{x}-v_{y}=35$. Then it is possible to estimate the coupling ratio at the normal operating point from the width of the resonance measured by detuning the horizontal tune. The other way is to extrapolate the coupling ratio from the Touschek lifetime.

The coupling ratio at the present operation point is $0.06 \%$ and the vertical emittance is smaller than $10^{-11} \mathrm{mrad}$ [3]. We can change the coupling ratios from $0.05 \%$ up to $80 \%$ only by changing the operation point in the tune diagram.

Direct measurement of the photon beam emittance and accordingly the electron beam emittance is underway. Preliminary results showed the vertical beam size is around $10 \mu \mathrm{m}$ or less.

\subsection{Orbit Stability}

The beam stability and reproducibility in position and angle is essential for the low emittance ring. The SPring8 storage ring and the beamlines are built on a stable rock to reduce a subsidence of the floor and ground vibrations induced outside of the SPring-8 campus. In addition, we have put a large effort to remove vibrations transmitted to the magnet girders from cooling-water pumps and to stabilize cooling water temperature. The digital feedback system is developed to stabilize the beam orbit within $0.7 \mu \mathrm{m}$ (in rms) in the horizontal plane and $0.4 \mu \mathrm{m}$ (in rms) in the vertical plane. Since the commissioning of the storage ring, a long-term variation of the circumference or the beam energy has been measured. There are two components, one with a period of 12 hours and the other with a period of one year. The former is well explained as induced by earth tide [4]. 


\subsection{Novel Insertion Devices}

High quality insertion devices are indispensable for the third generation synchrotron radiation source. We have developed various types of novel insertion devices. In Table 3 are listed the insertion devices which are currently operational and under construction. The in-vacuum undulator covers a very wide range of X-ray energy. For example, an undulator of $\lambda \mathrm{u}=32 \mathrm{~mm}$ provides $\mathrm{X}$-ray from $5.2 \mathrm{keV}$ to $18.5 \mathrm{keV}$ as the fundamental radiation, $15.5 \mathrm{keV}$ to $51 \mathrm{keV}$ as the $3^{\text {rd }}$ harmonics, and $26 \mathrm{keV}$ to $75 \mathrm{keV}$ as $5^{\text {th }}$ harmonics [5].

The helical undulator can provide circularly polarized Xray and has a special feature that only the fundamental radiation goes along the optical axis. Accordingly we have adopted a helical undulator as a source of the high-flux beamline. Figure- 8 undulator, on the contrary, can provide linearly polarized radiation [6]. It should be pointed out that integer-harmonic radiation is horizontally polarized but half-integer harmonics is vertically polarized. In addition to these we have developed an in-vacuum minipole undulator of $\lambda \mathrm{u}=11 \mathrm{~mm}$ for BNL, USA.

Table 3 List of Insertion Devices at SPring-8

\begin{tabular}{|l|c|l|c|}
\hline \multicolumn{2}{|c|}{ ID (operational) } & \multicolumn{2}{c|}{ ID (constructing) } \\
\hline \multicolumn{1}{|c|}{ Type of ID } & $\lambda \mathrm{u}(\mathrm{mm})$ & \multicolumn{1}{|c|}{ Type of ID } & $\lambda \mathrm{u}(\mathrm{mm})$ \\
\hline in-vacuum U* & 32 & in-vacuum U & 28 \\
& & & 32 \\
& & & 36 \\
& & & 40 \\
\hline tandem vertical U & 37 & hybrid in-vacuum U & 24 \\
\hline twin helical U & 120 & revolver type U & 40,100 \\
\hline figure-8 U & 120 & long U & 32 \\
& 26 & & 80 \\
\hline variably polar. U & 120 & & \\
\hline elliptical MPW & 120 & & \\
\hline
\end{tabular}

* U: undulator

** variably polarizing undulator

\section{NEW CAPABILITIES OF SPRING-8}

At the early stage of design work, we changed the original design of SPring-8 in the following way; we increased the electron energy from $6 \mathrm{GeV}$ to $8 \mathrm{GeV}$ and changed the magnet configuration of the storage ring from 48 -fold symmetric lattice to 4 -fold symmetric one with 4 long straight sections. At the same time we decided to leave a possibility to lengthen several beamlines by $300 \mathrm{~m}$ and $1000 \mathrm{~m}$. These modifications give new capabilities to SPring- 8 and some of them are being developed.

\subsection{Long Straight Section}

Main purpose of the long straight sections is to install a very long insertion device to get photons of higher brilliance. By now we have organized two international workshops to discuss technical issues related to long insertion divices as well as scope of science to be done with very brilliant $\mathrm{X}$-ray. Construction of the first beamline from a $25 \mathrm{~m}$-long undulator with $\lambda \mathrm{u}=32 \mathrm{~mm}$ starts in this year. At the same time a proposal to construct the second one is prepared. Design of two long undulators is in progress. Preliminary parameters are listed in Table 4.

The magnet arrangement in the long straight sections are determined. The emittance of this lattice is the same as that of the present lattice.

The magnet arrangement will be changed in the summer of 2000. Experimental program at this beamlines is being discussed.

Table 4 Design Parameters of Long Undulators

\begin{tabular}{|l|c|c|}
\hline & Long X-ray U & Long Helical U \\
\hline total length & $25 \mathrm{~m}$ & $25 \mathrm{~m}$ \\
\hline periodic length & $32 \mathrm{~mm}$ & $80 \mathrm{~mm}$ \\
\hline period number & 780 & 312 \\
\hline energy range & $\begin{array}{c}8 \sim 18 \mathrm{keV}\left(1^{\text {st }}\right) \\
24 \sim 38 \mathrm{keV}\left(3^{\text {rd }}\right) \\
40 \sim 50 \mathrm{keV}\left(5^{\text {th }}\right)\end{array}$ & $0.2 \sim 6 \mathrm{keV}\left(1^{\text {st }}\right)$ \\
\hline brilliance & $8 \times 10^{20}$ & $3 \times 10^{20}$ \\
\hline
\end{tabular}

\subsection{Long Beamline}

There are three beamlines which can be lengthened by $1,000 \mathrm{~m}$. The first one is from a standard-size undulator, the second is from a bending magnet, and the last is from a long undulator. Construction of one long beamline from the in-vacuum undulator with $\lambda \mathrm{u}=32 \mathrm{~mm}$ starts this year. The purpose of this beamline is to pursue various experiments with precise phase measurement in hard X-ray region. Experiments of ultra small angle scattering is also planned. The beamline will be completed in 2001 .

Two beamlines of $200 \mathrm{~m}$ long are under construction. One is a BM beamline and the other is an undulator beamline and both are used for medical application of synchrotron radiation. The former is in commissioning and test experiments will start in this fall. The latter will be completed in 2000. The experimental stations of these beamlines are in the building which locates $200 \mathrm{~m}$ apart from the experimental hall of SPring-8. 


\subsection{High Energy X-ray and Slow Positron Source}

Electron energy of $8 \mathrm{GeV}$ is very useful to produce high energy $\mathrm{X}$-ray in a $\mathrm{GeV}$ region through Compton back scattering. The Research Center for Nuclear Physics, Osaka University is constructing "Laser-Electron-Photon" beamline. In the first phase high power Ar gas laser will be used. The maximum energy in this case is $2.4 \mathrm{GeV}$ and the expected photon intensity is $5 \times 10^{6} / \mathrm{sec}$ at a stored current of $100 \mathrm{~mA}$. All photons are tagged by scattered electrons to determine their energy. The experiments will start in this fall.

Photons of energy higher than the threshold of $\mathrm{e}^{-} \mathrm{e}^{+}$pair production can produce slow positrons. The conventional way is to produce the positrons by bombarding high energy electrons on the metal target. On the other hand, if we can produce high-intensity photons of energy higher than $1 \mathrm{MeV}$, we will get slow positrons through $\mathrm{e}^{-} \mathrm{e}^{+}$pair production process in converter materials. This method has an advantage of making the source activation-free. We are collaborating with Budker Institute of Nuclear Physics to develop a 10T superconducting wiggler. Total yield of slow positrons at SPring-8 is estimated as $10^{10}-10^{11}$ from numerical simulations.

\subsection{Infrared Radiation}

A beamline for infrared spectroscopy is under construction at SPring-8. The advantage of using $8 \mathrm{GeV}$ electron beam is that more intensive infrared synchrotron radiation is emitted into narrow vertical angle from electrons in the orbit with the larger bending radius. We extract a very sharp infrared photon beam from the bent part of the dipole magnet and reflect it by copper mirror in horizontal direction. Expected energy range and photon number are 0.7 to $100 \mu \mathrm{m}$ and $10^{11}$ photons $/ \mathrm{sec} / 0.1 \%$ b.w at $10 \mu \mathrm{m}$, respectively.

\section{SUMMARY}

Machine studies and operation experience in the past two years show that the performance of SPring- 8 is remarkable. We expect that new projects to build the long undulator beamline, $1 \mathrm{~km}$ beamline and a slow positron source will open the new capability of the large scale synchrotron radiation source. It could be emphasized that high energy electron beam with ultra low emittance will create a new field of science and technology.

\section{REFERENCES}

[1] H.Kamitsubo, J.Synchrotron Rad. 5(1998) 162

[2] S.Date et al. "Operation and Performance of the SPring-8 Storage Ring" presented by H.Okuma at this Conference

[3] N.Kumagai et al. "Estimation of Betatron Coupling and Vertical dDispersion for SPring-8 Storage Ring" presented by M.Takao at this Conference

[4] S.Date and N.Kumagai, N.I.M. A421(1999) 417

[5] T.Tanaka and H.Kitamura, N.M.I. A364 (1995) 368 